\title{
Expression of Basic Helix-Loop-Helix Transcripts During Low Temperature Treatments in Grapevines
}

\author{
Seon Ae Kim ${ }^{1}$, Soon Young Ahn', Seung Heui Kim², Jeom Hwa Han', Hae Keun Yun ${ }^{1}$ * \\ ${ }^{1}$ Department of Horticulture and Life Science, Yeungnam University, Gyeongsan 712-749, Korea \\ ${ }^{2}$ National Institute of Horticultural and Herbal Science, RDA, Suwon 441-706, Korea
}

\begin{abstract}
The differential expression of six basic helix-loop-helix (bHLH) genes in response to low temperatures was studied by evaluating their mRNA levels in the buds and shoots of grapevines. Comparison of the amino acid sequences deduced from nucleotide sequences of the bHLH genes in Vitis labruscana transcripts revealed homologies ranging from 58.8\% (V1CEbHLH1 and VlCEbHLH4) to $8.2 \%$ (VvbHLH and VlCEbHLH3). Specific primers from unique regions of genes were obtained by alignment of nucleotide sequences and used to evaluate differential expression patterns of bHLH genes. The accumulation patterns of bHLH mRNAs were induced differentially and dependent on the treated temperatures, $-20^{\circ} \mathrm{C}$ in the buds and $-2^{\circ} \mathrm{C}$ and $4{ }^{\circ} \mathrm{C}$ in the shoots of ' $\mathrm{Campbell}$ Early' and 'Muscat Baily A' grapevines. The bHLH genes showed differential expression patterns in response to low temperatures. In 'Campbell Early', the expression of V1CEbHLH1, VlCEbHLH2, and VlCEbHLH5 was up-regulated, while VlCEbHLH3 was down-regulated in response to $-20^{\circ} \mathrm{C}$ cold stress. In 'Muscat Bailey A', the expression of all genes was rapidly up-regulated, peaking at $6 \mathrm{~h}$, then decreasing at $12 \mathrm{~h}$ after treatment.
\end{abstract}

Keywords Bud, Cold treatment, Transcription factor, Vitis labruscana

\section{INTRODUCTION}

Low temperature is a serious abiotic stress showing effects similar to drought, salinity, oxidative stress, and chemical toxicity. Cold stress, which includes chilling $\left(<20^{\circ} \mathrm{C}\right)$ and freezing $\left(<0^{\circ} \mathrm{C}\right)$ temperatures, adversely affects the growth and development of plants and significantly constrains the spatial distribution of plants and agricultural productivity. Some of the molecular and physiological changes that occur during cold acclimation are important to plant cold tolerance (Hsieh et al. 2004; Zhu et al. 2007).

Grape (Vitis spp.) is the most cultivated fruit crop in the world. Low temperature leads to a series of biochemical, molecular, and physiological changes in grape that cause adverse effects on their growth and production. Grapevines cultivated in cold regions during winter often suffer from various degrees of frost and freezing damage, which subsequently results in increased costs and limits further development of grape production (Zhang et al. 2013).
Basic helix-loop-helixes (bHLH) are generally involved in the responses to various abiotic stresses in higher plants and comprise one of the largest families of transcription factors (TFs) (Ledent and Vervoort 2001). Induction of bHLHs has been reported in carpel margin development of Arabidopsis (Heisler et al. 2001), anthocyanin synthesis of petunia (Spelt et al. 2000) and dahlia (Ohno et al. 2011), the jasmonate signaling pathway of rice (Seo et al. 2011), alkaloid biosynthesis of Coptis japonica (Yamada et al. 2011), and cold treatment of apple (Feng et al. 2012). However, the relationship of the participation and function of bHLH TFs to low temperature response still remains largely unknown.

In this study, bHLH transcripts with different expression levels upon transcriptome analysis of Vitis labruscana subjected to freezing temperatures were obtained. Nucleotide and deduced amino acid sequences were then compared by alignment of bHLH genes, and their differential expression in response to cold stress was analyzed in 'Campbell Early'

Received June 19, 2014; Revised June 25, 2014; Accepted June 27, 2014; Published June 30, 2014

*Corresponding author Hae Keun Yun, haekeun@ynu.ac.kr, Tel: +82-53-810-2942, Fax: +82-53-810-4659 
and 'Muscat Bailey A' grapevines.

\section{MATERIALS AND METHODS}

\section{Plant materials and cold treatment}

One-year-old vine cane with latent buds of 'Campbell Early' (moderately tolerant to cold stress) and 'Muscat Bailey A' (MBA, sensitive to cold stress) grapevines were harvested, cut to small pieces with one bud, and used for cold treatment. Cold treatment of buds was carried out at $-20^{\circ} \mathrm{C}$ in the dark for $0,6,12$, and $24 \mathrm{~h}$. Vines with green shoots were maintained under $16 / 8$-h photoperiod at $4^{\circ} \mathrm{C}$ for $0,1,2,3$, and 4 weeks and at $-2^{\circ} \mathrm{C}$ for $0,24,48,72$, and $96 \mathrm{~h}$. All samples were collected at the indicated times, immediately frozen in liquid nitrogen and stored at $-80^{\circ} \mathrm{C}$ until further use. Untreated samples were collected for use as a control. Five plants and 200 buds were used per treatment and each treatment was performed in triplicate.

\section{RNA extraction and cDNA synthesis}

Total RNA was extracted from grapevine buds using a modified version of the method described by Yang et al. (2008) and from grapevine leaves using a modified pine tree method described by Chang et al. (1993). The yield and quality of total RNA were measured based on the absorbance at 230, 260, and $280 \mathrm{~nm}$ using a Nano Drop spectrophotometer (ND-1000, Technologies Inc., USA). First-strand cDNA was synthesized from the total RNA (500 ng) using a GoScript ${ }^{\mathrm{TM}}$ reverse transcription system synthesis kit (Promega, Madison, USA) and subsequently used as a template for PCR.

\section{Cloning of bHLH genes and sequence analysis}

Among 35 DEGs identified upon transcriptome analysis of 'Campbell Early' treated with low temperature, six loci showing different expression level with more than 100 transcripts were selected for evaluation of their differential expression against low temperature. Specific primers of six bHLH genes were designed by alignment of the nucleotide sequences from the 'Campbell Early' transcripts and used to screen differential expression in response to low temperature of grapevines.

The ExPASY server was used to translate the nucleotide sequences into amino acid sequences (http://www.expasy.org). Nucleotide sequences were subjected to pairwise comparisons and multiple alignments of the deduced amino acid sequences were performed using the T-coffee (Notredame et al.

Table 1. Sequence of primers used for quantitative real-time PCR analysis.

\begin{tabular}{|c|c|c|}
\hline Name & $\mathrm{NABIC}^{\mathrm{z})}$ ID & Primer sequence \\
\hline \multirow[t]{2}{*}{ V1CEbHLH1 ${ }^{\mathrm{y})}$} & NS-0415-000001 & 5'-GGCACCAAAGACAAGAGCAAGT-3' \\
\hline & & 5'-GGTGTTGGCTTTTCCTGCATAC-3' \\
\hline \multirow[t]{2}{*}{ VlCEbHLH2 } & NS-0416-000001 & 5'-CCTTGCTGAAAGGGTCAGAAGA-3' \\
\hline & & 5'-CAAGCATTACTGCCTTCCCAGT-3' \\
\hline \multirow[t]{2}{*}{ VlCEbHLH3 } & NS-0417-000001 & 5'-TCCCGTCTTTGTTGTTCCCTTA-3' \\
\hline & & 5'-CAGTCTGATGGGGATGGATAGC-3' \\
\hline \multirow[t]{2}{*}{ VlCEbHLH4 } & NS-0418-000001 & 5'-AGAGAAGGGCAGGTGATGAAGA-3' \\
\hline & & 5'-CCGAGGATTTTCCTGTTCCTCT-3' \\
\hline \multirow[t]{2}{*}{ VlCEbHLH5 } & NS-0419-000001 & 5'-ATTCGAGCGAAAAGAGGATGTG-3' \\
\hline & & 5'-GCTTGTCCATGTTGGGAAAAAG-3' \\
\hline \multirow[t]{2}{*}{ VlCEbHLH6 } & NS-0420-000001 & 5'-CTCTGCAGTCAAACCAGCTGAA-3' \\
\hline & & 5'-ATGCAGCTCCACCTTTGTCTTC-3' \\
\hline
\end{tabular}

${ }^{7)}$ NABIC - National Agricultural Biotechnology Information Center, RDA, Korea.

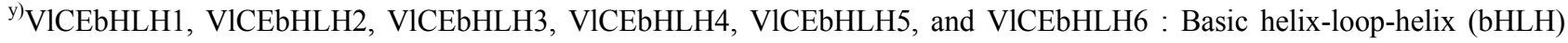
genes from Vitis labruscana cv 'Campbell Early'. 
2000). Tree nodes were evaluated by bootstrap analysis, and a single tree was constructed using the neighbor-joining method in MEGA 4.0 (Tamura et al. 2007). Nucleotide and amino sequence data of the VICEbHLH1, VlCEbHLH2, VlCEbHLH3, VlCEbHLH4, VlCEbHLH5, and VlCEbHLH6 were deposited in the National Agricultural Biotechnology Information Center (NABIC), Rural Development Administration, Korea database under the accession numbers NS-0415-000001, NS-0416-000001, NS-0417-000001, NS-0418-000001, NS-0419-000001, and NS-0420-000001, respectively.

\section{Quantitative real-time PCR analysis}

Real-time PCR was conducted on a C1000TM Thermal Cycler (CFX96TM Real-Time System, BioRad, CA, USA) using SYBR Premix Ex (SYBR Premix Ex Taq, TaKaRa Bio Inc., Osaka, Japan) as the fluorescent dye. Amplification was conducted by subjecting the samples to one cycle at $95^{\circ} \mathrm{C}$ for $30 \mathrm{~s}$, followed by 40 cycles of $95^{\circ} \mathrm{C}$ for $5 \mathrm{~s}$ and $60^{\circ} \mathrm{C}$ for $30 \mathrm{~s}$. Transcript levels were calculated using the standard-curve method and normalized against the grapevine actin gene (AB372563) as an internal control and non-treated samples as the reference sample, after which melting curves of the amplified products were recorded. For each gene, the reference sample was defined as the $1 \mathrm{x}$ expression level, and results were expressed as the fold increase of mRNA over the reference sample. All reactions were performed in triplicate to ensure consistency of the results.

\section{RESULTS}

Alignment of the amino acid sequences deduced from the nucleotide sequences of bHLH genes in $V$. labruscana transcripts is shown in Fig. 1. The homology of the amino acid sequence between each gene ranged from 58.8\% (VlCEbHLH1 and VlCEbHLH4) to $8.2 \%$ (VvbHLH and

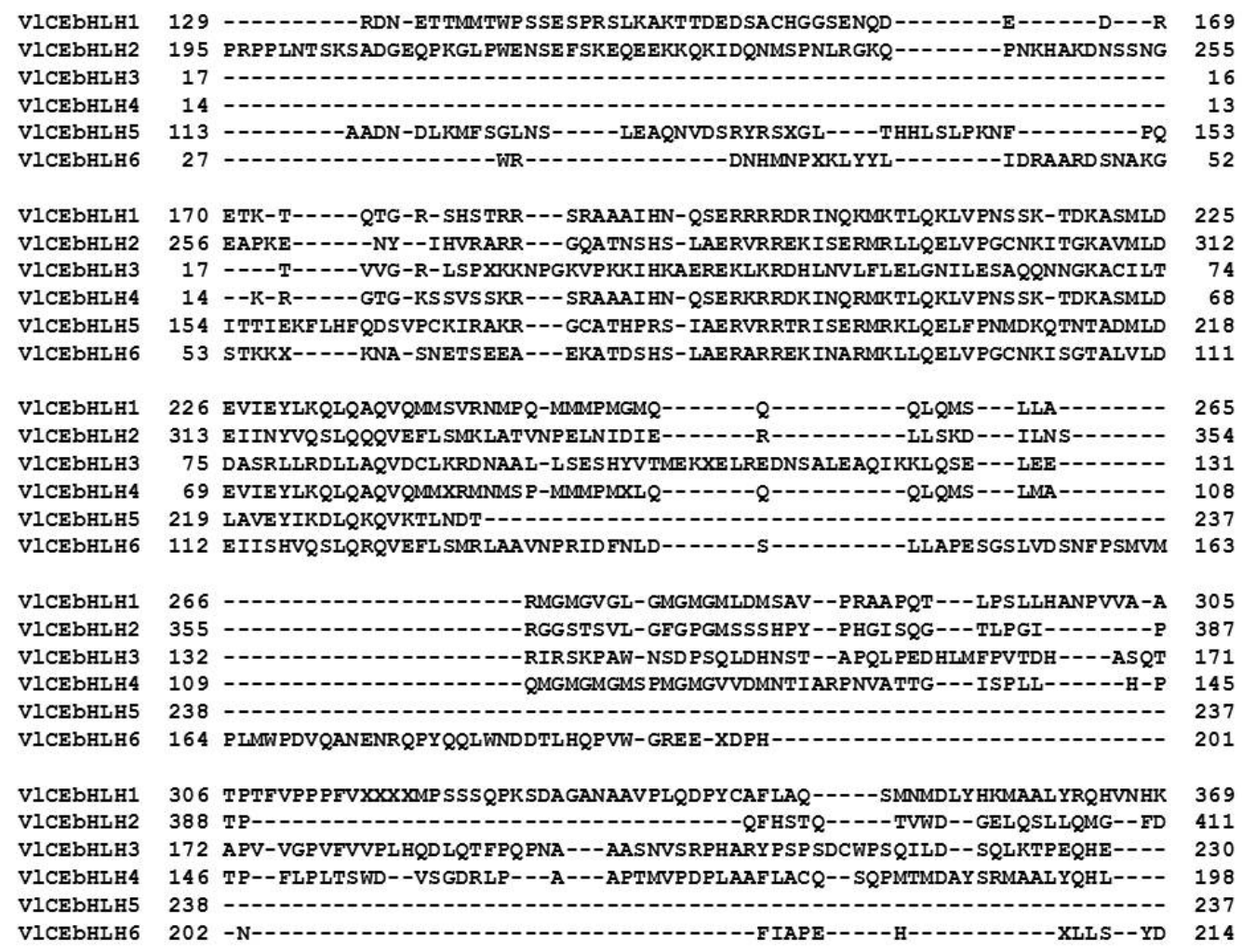

Fig. 1. Multiple amino acid sequence alignment of basic helix-loop-helix (bHLH). VlCEbHLH1 (NS-0415-000001), VlCEbHLH2 (NS-0416-000001), VlCEbHLH3 (NS-0417-000001), VlCEbHLH4 (NS-0418-000001), VlCEbHLH5 (NS-0419-000001), and VlCEbHLH6 (NS-0420-000001): bHLH proteins of Vitis labruscana cv. 'Campbell Early'. 
VlCEbHLH3). The amino acid sequences of VlCEbHLH2, VlCEbHLH5, VlCEbHLH6 and VlCEbHLH1 showed $39.6 \%, 29.3 \%, 26.5 \%$, and $25.2 \%$ homology with those of VlCEbHLH6, VlCEbHLH2, VlCEbHLH1 and VlCEbHLH5, respectively (Table 2). The resulting phylogenetic tree of the deduced amino acid sequence of bHLH transcripts is shown in Fig. 2. The bHLH sequences were divided into two groups, one including VvbHLH, VlCEbHLH2, VlCEbHLH5 and VlCEbHLH6, and another including VlCEbHLH1, VlCEbHLH3, and VlCEbHLH4.

Specific primers to each transcript were used to detect differential expression by real-time PCR of grapevine buds and leaves treated with various low temperatures. bHLH mRNAs showed differential expression patterns that were
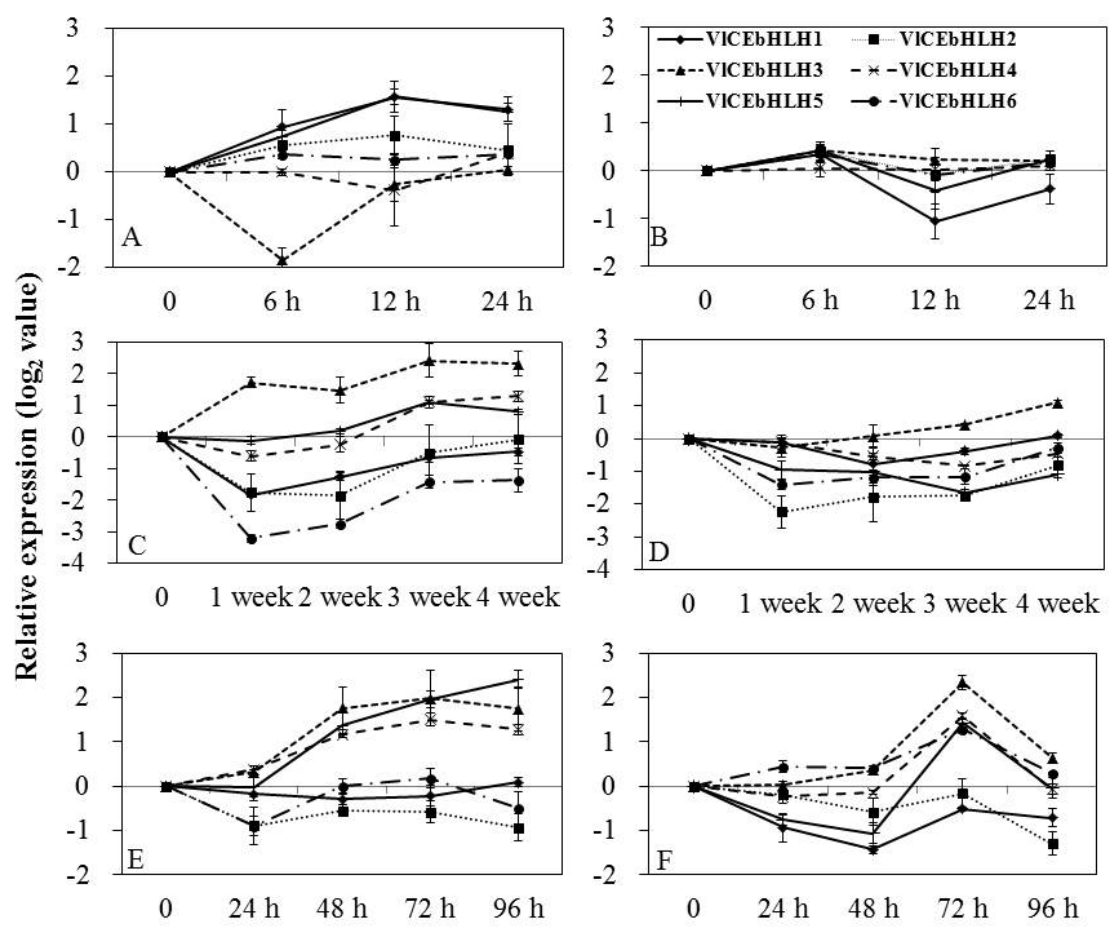

Fig. 2. Phylogenetic tree of basic helix-loop-helix (bHLH) proteins from VvbHLH (JQ823168.1) and Vitis labruscana transcripts, VlCEbHLH1 (NS-0415-000001), VlCEbHLH2 (NS-0416-000001), VlCEbHLH3 (NS-0417-000001), VlCEbHLH4 (NS-0418-000001), VlCEbHLH5 (NS-0419-000001), and VlCEbHLH6 (NS-0420-000001).

Table 2. Identity of amino acid sequences compared to basic helix-loop-helix (bHLH) gene transcripts of Vitis labruscana.

\begin{tabular}{cccccccc}
\hline \hline & VvbHLH $^{2)}$ & $\begin{array}{c}\text { VlCE } \\
\text { bHLH1 }\end{array}$ & $\begin{array}{c}\text { VlCE } \\
\text { bHLH2 }\end{array}$ & $\begin{array}{c}\text { VlCE } \\
\text { bHLH3 }\end{array}$ & $\begin{array}{c}\text { VlCE } \\
\text { bHLH4 }\end{array}$ & $\begin{array}{c}\text { VlCE } \\
\text { bHLH5 }\end{array}$ & $\begin{array}{c}\text { VlCE } \\
\text { bHLH6 }\end{array}$ \\
\hline VvbHLH & & 18.5 & 36.2 & 8.2 & 17.5 & 17.8 & 25.2 \\
VlCEbHLH1 & 18.5 & & 22.3 & 21.7 & 58.8 & 25.2 & 26.5 \\
VlCEbHLH2 & 36.2 & 22.3 & & 18.8 & 25.6 & 29.3 & 39.6 \\
VlCEbHLH3 & 8.2 & 21.7 & 18.8 & & 21.3 & 16.2 & 16.9 \\
VlCEbHLH4 & 17.5 & 58.8 & 25.6 & 21.3 & & 16.1 & 25.6 \\
VlCEbHLH5 & 17.8 & 25.2 & 29.3 & 16.2 & 16.1 & & 16.5 \\
VlCEbHLH6 & 25.2 & 26.5 & 39.6 & 16.9 & 25.6 & 16.5 & \\
\hline
\end{tabular}

${ }^{2)} \mathrm{VvbHLH}$ : originated from $V$. vinifera.

${ }^{\mathrm{y})} \mathrm{VlCEbHLH1,} \mathrm{VlCEbHLH2,} \mathrm{VlCEbHLH3,} \mathrm{VlCEbHLH4,} \mathrm{VlCEbHLH5,} \mathrm{and} \mathrm{VlCEbHLH6} \mathrm{:} \mathrm{bHLH} \mathrm{proteins} \mathrm{of} \mathrm{Vitis}$ labruscana cv 'Campbell Early'. 
dependent on various temperatures applied to 'Campbell Early' and 'MBA' (Fig. 3). In 'Campbell Early' buds treated with $-20^{\circ} \mathrm{C}$ cold stress (Fig. 3A), the expression of VlCEbHLH1, VlCEbHLH2, and VlCEbHLH5 was upregulated, peaking at $12 \mathrm{~h}$, while the expression of VlCEbHLH3 was down-regulated, peaking at $6 \mathrm{~h}$ after treatment. In MBA (Fig. 3B), the expression of all tested genes was rapidly up-regulated, peaking at $6 \mathrm{~h}$, then decreased at $12 \mathrm{~h}$ and increased again at $24 \mathrm{~h}$ after treatment. The mRNA level of VlCEbHLH3 in the leaves of grapevines maintained at $4^{\circ} \mathrm{C}$ (Fig. 3C and D), was up-regulated, while VlCEbHLH4 and VlCEbHLH5 were slightly down-regulated at one week, then increased again, peaking at four and three weeks after treatment. The mRNA level of VlCEbHLH1, VlCEbHLH2, and VlCEbHLH6 was down-regulated in 'Campbell Early' kept at $4{ }^{\circ} \mathrm{C}$, while the expression of VlCEbHLH1 and VlCEbHLH3 genes was down-regulated at one week and increased at four weeks in 'MBA' kept at $4^{\circ} \mathrm{C}$.

Following $-2^{\circ} \mathrm{C}$ cold treatment of the leaves of 'Campbell Early' grapevine, the expression of VlCEbHLH3, VlCEbHLH4, and VlCEbHLH5 was slightly increased at one hour, peaking at $72 \mathrm{~h}$ (VICEbHLH3 and VlCEbHLH4) and $96 \mathrm{~h}$ (VlCEbHLH5) after treatment (Fig. 3E). The accumulation of VlCEbHLH6 mRNA induced by cold stress $\left(-2^{\circ} \mathrm{C}\right)$ presented two peaks at $24 \mathrm{~h}$ and $72 \mathrm{~h}$ in 'MBA' grapevine (Fig. 3F). The VlCEbHLH1 and VlCEbHLH2 mRNA level were down-regulated and the VlCEbHLH3 mRNA level was up-regulated, peaking at $72 \mathrm{~h}$ after cold treatment $\left(-2^{\circ} \mathrm{C}\right)$.

\section{DISCUSSION}

Among various environmental stresses, low temperature is one of the most important factors limiting cultivation of plants. bHLH protein comprises a group of functionally diverse transcription factors found in plants. In the present study, six bHLH genes showed differential expression in response to various low temperatures that caused physiological damage and low productivity in grapevines. To examine the expression patterns of 'Campbell Early' bHLH genes, primers specific for unique regions in each gene were obtained using multiple sequence alignments. Quantitative PCR revealed that six bHLH genes were differently expressed in 'Campbell Early' transcripts.

Among the TFs, the bHLH motif-containing TFs are important regulatory components of the transcriptional networks. Expression of bHLH genes related to low temperature has been identified in various plants, including

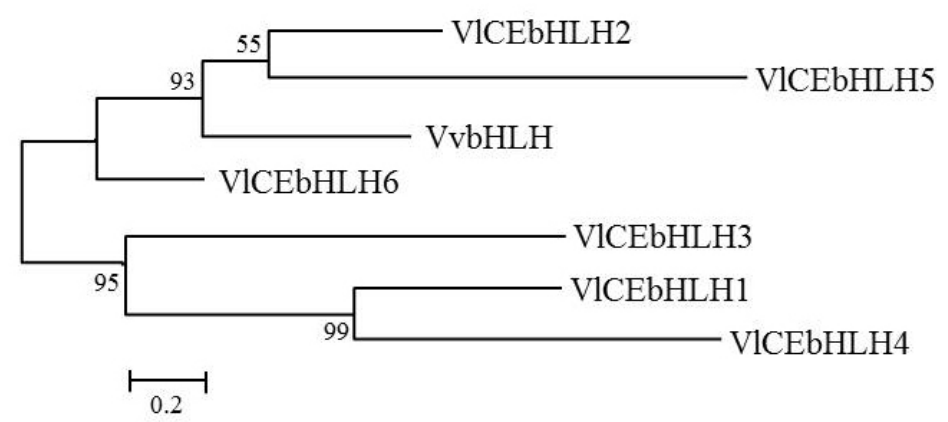

Fig. 3. Expression of basic helix-loop-helix (bHLH) under various low temperatures in grapevines. Transcript levels were calculated using the standard curve method from triplicate data with the grapevine actin gene as an internal control and non-treated bud and leaves (at time zero) as a reference. The results represent the mean fold increase in mRNA level over non-treated leaves relative to the $1 \mathrm{x}$ expression level. Results are the means of triplicate data from three experiments. Vertical bars indicate the standard deviation of three replicates. A and B, expression following freezing treatment $\left(-20^{\circ} \mathrm{C}\right)$ of buds for $0,6,12$ and $24 \mathrm{~h} ; \mathrm{C}$ and $\mathrm{D}$, expression following low treatment $\left(4^{\circ} \mathrm{C}\right)$ of shoots for $0,1,2,3$, and 4 weeks; $\mathrm{E}$ and $\mathrm{F}$, expression following freezing treatment $\left(-2^{\circ} \mathrm{C}\right)$ of shoots for 0, 24, 48, 72, and $96 \mathrm{~h}$. A, C, and E, 'Campbell Early'; B, D, and F, 'Muscat Baily A'. VlCEbHLH1, VlCEbHLH2, VlCEbHLH3, VlCEbHLH4, VlCEbHLH5, and VlCEbHLH6 : bHLHs genes from Vitis labruscana cv 'Campbell Early'. 
Arabidopsis (Penfield et al. 2005), apple (Xie et al. 2012), and Poncirus trifoliata (Huang et al. 2013). Xie et al. (2012) reported that apple bHLH TF MdbHLH3 is induced to regulate anthocyanin accumulation and fruit coloration at low temperature.

OsbHLH1 can be induced by low temperature and has been implicated in rice cold defense through an abscisic acid (ABA)-independent pathway (Wang et al. 2003). Up-regulation of the OsbHLH148 transcript level by low temperature resulted in activation of tolerance to drought (Seo et al. 2011). Feng et al. (2012) reported that the apple bHLH gene, MdCIbHLH1 (Cold-Induced bHLH1), encodes an ICE-like protein, acts as a signal transduction component in the CBF pathway and is associated with cold tolerance in apple. Additionally, overexpression of MdCIbHLH1 enhanced cold tolerance in transgenic Arabidopsis. In Arabidopsis thaliana plants, ICE2, which is a bHLH transcription factor and positive regulator, participates in the response to freezing through a cold acclimation-dependent pathway (Fursova et al. 2009). Although several bHLH genes were up-regulated by coldness, the transcript level of CsICE1 in Camellia sinensis was not altered by chilling treatment (Wang et al. 2012). These reports indicate that the bHLH genes respond differentially to abiotic stimuli in many plant models.

Buck and Atchley (2003) described 118 and 131 potentially unique bHLH proteins found in the A. thaliana and the Oryza sativa genomes, respectively. bHLH proteins are the second largest class of plant TFs, with 162 members in Arabidopsis and 167 members in rice (Carretero-Paulet et al. 2010; Feller et al. 2011). Most bHLH proteins identified were functionally characterized in model plants such as Arabidopsis and rice, and their roles include regulation of phytochrome signaling, flavonoid biosynthesis, fruit dehiscence, carpel, anther and epidermal cell development, hormone signaling and stress responses (Feller et al. 2011).

In this study, we analyzed nucleotide sequences and the amino acid sequences deduced from these nucleotides and compared the homologies among six bHLHs from $V$. labruscana. Additionally, we investigated the expression pattern of bHLH genes in response to various low temperature treatments in 'Campbell Early' and 'MBA' grapevines. The expression of bHLH transcripts differed following cold treatment, suggesting that the bHLH gene may be involved in cold stress response of grapevine. Identification of the differential expression of the six bHLH transcripts tested in this study in response to different cold conditions will facilitate elucidation of mechanisms of tolerance to coldness, improvement of freezing tolerance, and development of critical molecular markers for selection of cold-hardy seedlings in grapevine breeding programs.

\section{ACKNOWLEDGMENT}

This work was supported by a grant (No. PJ008224) from the Agricultural R\&D Project, Rural Development Administration, Republic of Korea.

\section{REFERENCES}

Buck MJ, Atchley WR. 2003. Phylogenetic analysis of plant basic helix-loop-helix proteins. J. Mol. Evol. 56: 742750 .

Carretero-Paulet L, Galstyan A, Roig-Villanova I, MartinezGarcia JF, Bilbao-Castro JR, Robertson DL. 2010. Genome-wide classification and evolutionary analysis of the bHLH family of transcription factors in Arabidopsis, poplar, rice, moss, and algae. Plant Physiol. 153: 13981412.

Chang S, Puryear J, Cairney J. 1993. A simple and efficient method for isolating RNA from pine trees. Plant Mol. Biol. 11: 113-116.

Feller A, Machemer K, Braun EL, Grotewold E. 2011. Evolutionary and comparative analysis of MYB and bHLH plant transcription factors. Plant J. 66: 94-116.

Feng XM, Zhao Q, Zhao LL, Qia Y, Xie XB, Li HF, Yao YX, You CX, Hao YJ. 2012. The cold-induced basic helix-loop-helix transcription factor gene $M d C I b H L H 1$ encodes an ICE-like protein in apple. BMC Plant Biol. 12: 22 .

Fursova OV, Pogorelko GV, Tarasov VA. 2009. Identification of ICE2, a gene involved in cold acclimation which determines freezing tolerance in Arabidopsis thaliana. Gene 429: 98-103.

Heisler MG, Atkinson A, Bylstra YH, Walsh R, Smyth DR. 
2001. SPATULA, a gene that controls development of carpel margin tissues in Arabidopsis, encodes a bHLH protein. Development 128: 1089-1098.

Hsieh TH, Lee JT, Yang PT, Chiu LH, Charng YY, Wang YC, Chan MT. 2004. Heterology expression of the Arabidopsis C-repeat/dehydration response element binding factor 1 gene confers elevated tolerance to chilling and oxidative stresses in transgenic tomato. Plant Physiol. 135: 1145-1155.

Huang XS, Wang W, Zhang Q, Liu JH. 2013. A basic helix-loop-helix transcription factor, PtrbHLH, of Poncirus trifoliata confers cold tolerance and modulates peroxidasemediated scavenging of hydrogen peroxide. Plant Physiol. 162: 1178-1194.

Ledent V, Vervoort M. 2001. The basic helix-loop-helix protein family: comparative genomics and phylogenetic analysis. Genome Res. 11: 754-770.

Notredame C, Higgins DG, Heringa J. 2000. T-Coffee: A novel method for fast and accurate multiple sequence alignment J. Mol. Biol. 302: 205-217.

Ohno S, Hosokawa M, Hoshino A, Kitamura Y, Morita Y, Park KI, Nakashima A, Deguchi A, Tatsuzawa F, Doi M, Iida S, Yazawa S. 2011. A bHLH transcription factor, DvIVS, is involved in regulation of anthocyanin synthesis in dahlia (Dahlia variabilis). J. Exp. Bot. 62: 5105-5116.

Penfield S, Josse EM, Kannangara R, Gilday AD, Halliday KJ, Graham IA. 2005. Cold and light control seed germination through the bHLH transcription factor SPATULA. Current Biol. 15: 1998-2006.

Seo JS, Joo J, Kim MJ, Kim YK, Nahm BH, Song SI, Cheong JJ, Lee JS, Kim JK, Choi YD. 2011. OsbHLH148, a basic helix-loop-helix protein, interacts with OsJAZ proteins in a jasmonate signaling pathway leading to drought tolerance in rice. Plant J. 65: 907-921.

Spelt C, Quattrocchio F, Mol JN, Koes R. 2000. Anthocyanin1 of petunia encodes a basic helix-loop-helix protein that directly activates transcription of structural anthocyanin genes. Plant Cell 12: 1619-1632.

Tamura K, Dudley J, Nei M, Kumar S. 2007. MEGA4: Molecular evolutionary genetics analysis (MEGA) software version 4.0. Mol. Biol. Evol. 24: 1596-1599.

Wang Y, Jiang CJ, Li YY, Wei CL, Deng WW. 2012. CsICE1 and $\mathrm{CsCBF}$ 1: two transcription factors involved in cold responses in Camellia sinensis. Plant Cell Rep. 31: 27-34.

Wang YJ, Zhang ZG, He XJ, Zhou HL, Wen YX, Dai JX, Zhang JS, Chen SY. 2003. A rice transcription factor OsbHLH1 is involved in cold stress response. Theor. Appl. Genet. 107: 1402-1409.

Xie XB, Li S, Zhang RF, Zhao J, Chen YC, Zhao Q, Yao YX, You CX, Zhang XS, Hao YJ. 2012. The bHLH transcription factor MdbHLH3 promotes anthocyanin accumulation and fruit colouration in response to low temperature in apples. Plant Cell Environ. 35: 1884-1897.

Yamada Y, Kokabu Y, Chaki K, Yoshimoto T, Ohgaki M, Yoshida S, Kato N, Koyama T, Sato F. 2011. Isoquinoline alkaloid biosynthesis is regulated by a unique bHLH-type transcription factor in Coptis japonica. Plant Cell Physiol. 52: 1131-1141.

Yang G, Zhou R, Tang T, Shi S. 2008. Simple and efficient isolation of high-quality total RNA from Hibiscus tiliaceus, a mangrove associate and its relatives. Prep. Biochem. Biotechnol. 38: 257-264.

Zhang J, Liu N, Niu R, Liu Y, Zhai H, Xu W, Wang Y. 2013. Construction of a cDNA library of the Chinese wild Vitis amurensis under cold stress and analysis of potential hardiness-related expressed sequence tags. Genet. Mol. Res. 12: 1182-1193.

Zhu JH, Dong CH, Zhu JK. 2007. Interplay between coldresponsive gene regulation, metabolism and RNA processing during plant cold acclimation. Curr. Opin. Plant Biol. 10: 290-295. 